\title{
AMCoR
}

Asahikawa Medical University Repository http://amcor.asahikawa-med.ac.jp/

Biochemical and biophysical research communications (2013.Feb) 431 巻1号:25 30.

Endoplasmic reticulum stress suppresses lipin-1 expression in 3T3-L1 adipocytes

Takahashi N, Yoshizaki T, Hiranaka N, Suzuki T, Yui T, Akanuma M, Kanazawa K, Yoshida M, Naito S, Fujiya M, Kohgo $\mathrm{Y}$, Ieko M 


\section{Endoplasmic reticulum stress suppresses lipin-1 expression in 3T3-L1 adipocytes}

Nobuhiko Takahashi $^{\mathrm{a}, \mathrm{b}, *}$, Takayuki Yoshizaki ${ }^{\mathrm{c}}$, Natsumi Hiranaka ${ }^{\mathrm{a}}$, Takeshi Suzuki ${ }^{\mathrm{a}}$, Tomoo Yuid ${ }^{\mathrm{d}}$, Masayasu Akanuma ${ }^{\mathrm{d}}$, Kaoru Kanazawa ${ }^{\mathrm{e}}$, Mika Yoshida ${ }^{\mathrm{f}}$, Sumiyoshi Naito $^{\mathrm{f}}$, Mikihiro Fujiya ${ }^{\mathrm{b}}$, Yutaka Kohgo ${ }^{\mathrm{b}}$, and Masahiro Ieko ${ }^{\mathrm{a}}$

${ }^{a}$ Department of Internal Medicine, School of Dentistry, Health Sciences University of Hokkaido, 1757 Kanazawa, Ishikari-Toubetsu, Hokkaido 061-0023, Japan

${ }^{b}$ Division of Gastroenterology and Hematology/Oncology, Department of Medicine, Asahikawa Medical University, 2-1-1-1 Midorigaoka-Higashi, Asahikawa, Hokkaido 078-8510, Japan

'Innovation Center, Kagoshima University, 1-21-40 Korimoto, Kagoshima 890-0065, Japan

${ }^{\mathrm{d}}$ Department of Fixed Prosthodontics and Oral Implantology, School of Dentistry, Health Sciences University of Hokkaido, 1757 Kanazawa, Ishikari-Toubetsu, Hokkaido 061-0023, Japan

${ }^{\mathrm{e}}$ Department of Dental Anesthesiology, School of Dentistry, Health Sciences University of Hokkaido, 1757 Kanazawa, Ishikari-Toubetsu, Hokkaido 061-0023, Japan

${ }^{\mathrm{f}}$ Department of Clinical Laboratory, Health Sciences University of Hokkaido, 1757 Kanazawa, Ishikari-Toubetsu, Hokkaido 061-0023, Japan 
*Corresponding author: Nobuhiko Takahashi, MD, PhD

Department of Internal Medicine, School of Dentistry, Health Sciences University of

Hokkaido, 1757, Kanazawa, Ishikari-Toubetsu, Hokkaido 061-0023, Japan

Telephone/Fax: +81133231534

E-mail address: ntkhs@hoku-iryo-u.ac.jp (N.Takahashi). 


\begin{abstract}
Lipin-1 plays crucial roles in the regulation of lipid metabolism and cell differentiation in adipocytes. In obesity, adipose lipin-1 mRNA expression is decreased and positively correlated with systemic insulin sensitivity. Amelioration of the lipin-1 depletion might be improved dysmetabolism. Although some cytokines such as TNF- $\alpha$ and interleukin-1 $\beta$ reduces adipose lipin- 1 expression, the mechanism of decreased adipose lipin-1 expression in obesity remains unclear. Recently, endoplasmic reticulum (ER) stress is implicated in the pathogenesis of obesity. Here we investigated the role of ER stress on the lipin-1 expression in 3T3-L1 adipocytes. We demonstrated that lipin-1 expression was suppressed by the treatment with ER stress inducers (tunicamycin and thapsigargin) at transcriptional level. We also showed that constitutive lipin-1 expression could be maintained by peroxisome proliferator-activated receptor- $\gamma$ in 3T3-L1 adipocytes. Activation of peroxisome proliferator-activated receptor- $\gamma$ recovered the ER stress-induced lipin-1 suppression. These results suggested that ER stress might be involved in the pathogenesis of obesity through lipin-1 depletion.
\end{abstract}

Keywords: Lipin-1; Adipocyte; Endoplasmic reticulum stress; Obesity 


\section{Introduction}

Lipin-1 was originally identified by positional cloning as the mutated gene in fatty liver dystrophy (fld) mice characterized by lipodystrophy, neonatal fatty liver, and peripheral neuropathy [1]. Three lipin family genes including lipin-1, lipin-2, and lipin-3 have been found, and exhibit distinct tissue-specific expression patterns [2]. In adipocytes, lipin-1 is expressed at high levels, whereas lipin-2 is presented at low levels in mice [2]. The function of lipin-1 has been revealed in the diverse regulation of metabolism. First, lipin-1 promotes triglyceride synthesis as an enzyme, phosphatidic acid phosphatase, which catalyzes the formation of diacylglycerol from phosphatidic acid [3]. Second, lipin-1 is involved in adipogenesis and maintenance of adipocyte function to facilitate peroxisome proliferator-activated receptor (PPAR)- $\gamma$ expression $[4,5]$. Third, lipin-1 regulates lipid metabolism as a co-activator interacting with several transcription factors (e.g. PPAR- $\alpha$ ) or co-activator protein (PPAR- $\gamma$ coactivator-1 $\alpha$ ) [6]. Last, lipin-1 regulates inflammation in adipocyte by acting as a co-repressor to suppress the expression of pro-inflammatory cytokines [7]. Taken together, these findings establish lipin-1 as a multiple regulator of metabolism.

Lipin-1 expression levels in adipose tissue have been of great interest for investigation, because the alteration of lipin-1 gene expression causes lipodystrophy phenotype in mice. The investigations revealed that adipose lipin-1 mRNA expression levels are positively correlated with insulin sensitivity in the subject with obesity, or in healthy people. The expression levels are negatively correlated with adiposity, or the presence of impaired glucose tolerance or the metabolic syndrome 
[8-13]. The reduced expression levels of lipin-1 in adipose tissue can be recovered its levels by weight reduction $[12,13]$. Moreover, adipocyte-specific lipin-1 transgenic mice fed a high-fat diet developed obesity but unexpectedly showed an amelioration in insulin resistance [14]. These in vivo studies substantiated that adipose lipin-1 expression levels are positively correlated with favorable for overall insulin sensitivity. The significance of depleted adipose lipin-1 expression in obesity has been partially determined. Kim et al. reported the forced depletion of lipin-1 in adipocytes induces TNF- $\alpha$ production [7]. We previously have reported that the knockdown of lipin-1 in 3T3-L1 adipocytes resulted in the induction of monocyte chemoattractant protein-1 [15]. These lines of evidence established that the depleted adipose lipin-1 expression promotes adipose inflammation. Therefore, understanding the mechanism of reduced adipose lipin-1 expression in obesity is important for providing the new treatment of metabolic diseases, including obesity, the metabolic syndrome and type 2 diabetes.

During the development of obesity, cells are evoked by various chemical and physical stresses. Recently endoplasmic reticulum (ER) stress has emerged as an important mediator in the pathogenesis of obesity and diabetes, especially observed in pancreatic $\beta$-cell, liver, muscle, and hypothalamus in obesity [16]. Moreover, recent studies have demonstrated the importance of ER stress in the adipose tissue of obese rodents and humans [17-21], induced by various stimuli such as tissue hypoxia, free fatty acids, and mitochondria uncoupling [22-24]. We therefore hypothesized that the ER stress may be involved in lipin-1 depletion in adipocytes. 


\section{Materials and methods}

\subsection{Cell culture and adipocyte differentiation}

3T3-L1 fibroblasts were purchased from the American Type Culture Collection (Manassas, VA, USA). For each experiment, cells were seeded into 6-well culture plates. The cells were induced to differentiate into adipocytes according to the standard protocol as described previously [15]. Fully differentiated adipocytes were used at 12-14 days after induction of differentiation.

\subsection{Cell treatment}

Tunicamycin and thapsigargin were purchased from Sigma (St. Louis, MO, USA), and dissolved with DMSO to make a stock solution. ER stress was induced chemically by the treatment of $3 \mu \mathrm{g} / \mathrm{ml}$ tunicamycin, and $50 \mathrm{nM}$ thapsigargin for 24 $\mathrm{h}$ into fully differentiated adipocytes unless otherwise specified. For analyzing the mechanism of thapsigargin-induced lipin-1 mRNA reduction, a transcription inhibitor, actinomycin D (Sigma) or DMSO was co-treated with thapsigargin into 3T3-L1 adipocytes. To assess the involvement of PPAR- $\gamma$ in lipin-1 expression in adipocytes, cells were treated with a PPAR- $\gamma$ agonist, ciglitazone (Merck, Darmstadt, Germany). DMSO was used as vehicle adjusted at a final concentration of $0.1 \%$ in the medium throughout the experiments. 


\subsection{Quantitative real-time PCR analysis}

RNA extraction and cDNA synthesis were performed as described previously [15]. Briefly, quantitative real-time PCR analysis were performed with an Applied Biosystems 7500 Sequence Detection System using TaqMan Gene Expression master mix, according to the manufacturer's instructions (Applied Biosystems, Foster City, CA, USA). Validated TaqMan Gene Expression Assays containing gene specific TaqMan probes and primers (Applied Biosystems) for lipin-1 (Mm00550511_m1), lipin-2 (Mm00522390_m1),

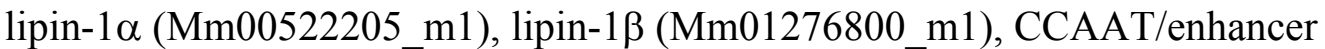
binding protein (C/EBP)- $\alpha\left(M m 00514283 \_s 1\right)$, and PPAR- $\gamma\left(M m 01184322 \_m 1\right)$ were used for assay-on-demand gene expression products. Amplification was determined using the comparative threshold cycle $(\mathrm{Ct})$ method and Sequence Detection Software version 1.4 (Applied Biosystems). The $2^{-\mathrm{ddCt}}$ method was used to calculate the relative expression of mRNA [15]. Results are normalized by the expression levels of eukaryotic 18S rRNA gene (Hs99999901_s1) and then calculated as fold change relative to the vehicle control. All experiments were performed at least in triplicate.

\subsection{Protein extraction and Western blotting}

Protein extraction and Western blotting were performed as described previously [15]. In brief, equal amounts of extracted protein $(20-50 \mu \mathrm{g}$ per lane) 
were resolved on SDS-PAGE for Western blotting using antibodies against mouse lipin-1, BiP, CHOP (Cell Signaling technologies, Danvers, MA, USA), C/EBP- $\alpha$, PPAR- $\gamma$, and $\beta$-actin (Santa Cruz Biotechnology, Santa Cruz, CA, USA). The bands were visualized with a Light Capture II system (ATTO Co., Tokyo, Japan).

2.4. Gene silencing by small interfering RNA (siRNA)

Predesigned siRNA against C/EBP- $\alpha$ (MSS273621), PPAR- $\gamma$ (MSS207863) and negative control (NC) siRNA (Stealth ${ }^{\mathrm{TM}}$ RNAi Negative Control Low GC Duplex \#2) were purchased from Invitrogen (Carlsbad, CA, USA).

Transfection into 3T3-L1 adipocytes was performed by lipofection using Lipofectamine RNAiMAX (Invitrogen) according to the manufacturer's instruction with siRNA at a final concentration of $50 \mathrm{nM}$. The cells were examined at $60 \mathrm{~h}$ after the transfection.

\subsection{Statistical Analysis}

The results are expressed as means $\pm \mathrm{SE}$. For comparison of more than two groups, ANOVA was performed followed by Turkey's multiple comparison tests

using GraphPad PRISM software version 5 (GraphPad Software Inc., San Diego, CA, USA). Two-tailed unpaired Student's $t$-tests were used for comparison of the two groups. $P<0.05$ was considered statistically significant. 


\section{Results}

3.1. Lipin-1 expression is reduced by ER stress activation in 3T3-L1 adipocytes

We first treated 3T3-L1 adipocytes with tunicamycin (an inhibitor of $\mathrm{N}$-glycosylation), and thapsigargin (an inhibitor of the ER $\mathrm{Ca}^{2+}$-ATPase), both of which caused ER stress (Supplementary Fig. S1). Then, lipin-1 expression levels were evaluated in 3T3-L1 adipocytes under ER stress. As shown in Fig. 1A-C, the treatment with tunicamycin and thapsigargin reduced lipin-1 mRNA expression in dose- and time-dependent manners. Protein expression of lipin-1 was also reduced by the treatment with both ER stress inducers (Fig. 1D). The two lipin-1 splice isoforms, lipin-1A and lipin-1B, are identified in adipocytes. Fig. 1E showed that the treatment reduced both lipin-1A and $-1 \mathrm{~B}$ with a greater effect on lipin-1B. As illustrated in Fig. 1F, mRNA expression levels of lipin-2 were not altered by the ER stress induction. These results indicated that ER stress induction reduces lipin-1 expression in 3T3-L1 adipocytes.

\subsection{ER stress-induced lipin-1 depletion is regulated at transcriptional level}

To determine whether the depletion of lipin-1 mRNA levels by ER stress is due to alterations in mRNA stability, actinomycin D was used to inhibit the synthesis of new mRNA molecules, and then the degradation of existing lipin-1 mRNA in the 
treatment with either thapsigargin or vehicle was compared. As demonstrated in Fig. 2, the decline of lipin-1 mRNA levels was similar in both treatment groups, indicating that mRNA destabilization was not involved in the reduction of lipin-1 transcripts by thapsigargin. This result led us to believe that the lipin-1 depletion by ER stress is regulated at the transcriptional level.

\subsection{PPAR- $\gamma$ could be involved in the constitutive expression of lipin-1}

Data are lacking on the regulators of constitutive lipin-1 expression in adipocytes. Two transcription factors, C/EBP- $\alpha$ and PPAR- $\gamma$, are well documented in adipocyte functions. Because these transcription factors are reduced by ER stress in adipocytes [25], these molecules might be involved in the constitutive expression of lipin-1. We first confirmed that C/EBP- $\alpha$ and PPAR- $\gamma$ were markedly reduced in both protein (Fig. 3A) and mRNA (Fig. 3B and C) levels by the treatment with tunicamycin and thapsigargin. Then, the gene expression of C/EBP- $\alpha$ (Fig. 3D) or PPAR- $\gamma$ (Fig. 3E) was significantly knocked down using specific siRNA in 3T3-L1 adipocytes. Knockdown of PPAR- $\gamma$ expression resulted in the significant reduction of lipin-1 expression by 44\% (Fig. 3F), whereas knockdown of C/EBP- $\alpha$ did not altered the expression. These results suggested that PPAR- $\gamma$ could be involved in the constitutive expression of lipin-1 in 3T3-L1 adipocytes.

3.4. Ciglitazone, a selective PPAR- $\gamma$ agonist, recovered the ER stress-induced lipin-1 depletion 
Next we assessed the involvement of PPAR- $\gamma$ in the thapsigargin-induced suppression of lipin-1 expression in 3T3-L1 adipocytes. 3T3-L1 adipocytes were treated with thapsigargin or a vehicle for $24 \mathrm{~h}$, and then a selective PPAR- $\gamma$ agonist, ciglitazone, or a vehicle was co-treated in the medium during the last $8 \mathrm{~h}$ of the treatment. The treatment of ciglitazone increased the lipin-1 mRNA (Fig. 4A) and protein levels (Fig. 4B), and also recovered the reduced expression levels of thapsigargin-induced lipin-1 to the control levels. PPAR- $\gamma$ protein expression was not increased by the treatment of ciglitazone (Fig. 4C), The protein expression levels of BiP and CHOP, typical ER stress markers, were not decreased by the treatment of ciglitazone (Fig. 4C).

\section{Discussion}

We have shown that ER stress, which develops in obesity, suppresses lipin-1 mRNA and protein expression in 3T3-L1 adipocytes, presumably through the involvement of PPAR- $\gamma$. So far only one study has mentioned the relation between ER stress and lipin-1 expression in adipose tissue. Miranda et al. analyzed lipin-1 gene expression in parallel with several hypoxia, angiogenic, ER stress and PPAR-related genes in the adipose tissues of 62 human obese subjects [26]. They found a positive correlation between some ER stress genes (CHOP10, XBP1, and HSP90) and lipin-1 gene expression levels using Pearson's correlation analysis in an 
opposite fashion to our results. The reason for this discrepancy may be explained by differences in study design. We observed direct responses to ER stress in adipocytes, whereas they observed the correlation of gene expression in adipose tissue including adipocytes, fibroblasts, vascular endothelial cells, and immune cells. In developing obesity, increased ER stress is observed in adipocytes, and also decreased lipin-1 expression is well established. Our results that ER stress reduces lipin-1 expression in adipocytes are in reasonable agreement with this notion.

Lipin-1 consists of three isoforms, lipin-1 $\alpha$ (1A), lipin-1 $\beta$ (1B), and lipin- $1 \gamma$ [27]. Induction of ER stress reduced the expression of both lipin-1A and lipin-1B isoforms, albeit with greater effect on lipin-1B (Fig. 1E). In adipocytes, studies with these lipin-1 isoforms characterized that lipin-1A is localized mainly in nucleus and induces adipogenic genes, whereas lipin-1B is localized mainly in cytosol and induces lipogenic genes [28]. Because ER is located in cytosol and is the site of triacylglycerol synthesis, the prominent decrease in lipin-1B expression by ER stress might be relevant to ER functions, although it requires further investigation.

In liver, ER stress induces lipin-2 expression thereby promoting hepatic insulin resistance via the modulation of hepatic lipid composition [29]. Lipin-2 is expressed in adipocytes, even though less abundant than lipin-1. We therefore tested whether ER stress induction affects lipin-2 expression in adipocytes. The result showed that ER stress did not alter lipin-2 expression (Fig. 1F), suggesting the role of ER stress in the expression of lipin family genes is in a tissue-dependent manner.

The data presented in Figure 2 strongly suggest that the mechanism of lipin-1 depletion induced by ER stress is occurred at the transcriptional level. Although some transcription factors such as C/EBP- $\alpha$, PPAR- $\gamma$, and glucocorticoid 
receptor are involved in the transcriptional activation of lipin-1 gene in adipocytes [4, 30], not much has been clarified the transcription factor controlling the constitutive expression of lipin-1 in adipocytes. On the other hand, the expression of C/EBP- $\alpha$ and PPAR- $\gamma$ has been shown to decrease by ER stress in 3T3-L1 adipocytes [25]. We therefore made an assumption that these transcriptional factors might be involved in the constitutive lipin-1 gene expression in adipocytes. Our present results provide evidence that PPAR- $\gamma$ can be involved in the constitutive expression of lipin-1 in fully differentiated adipocytes. The observation that lipin-1 mRNA levels were positively correlated with PPAR- $\gamma$ mRNA levels in human adipose tissue may support our result [9].

We next tested whether the activation of PPAR- $\gamma$ ameliorates the reduced lipin-1 expression by ER stress. The treatment with ciglitazone, a PPAR- $\gamma$ agonist, recovered the ER stress-induced depletion of lipin-1 expression to the control levels (Fig. 4A and B). The protein expression of PPAR- $\gamma$ was not increased by the treatment of ciglitazone (Fig. 4C). Although pioglitazone, a PPAR- $\gamma$ agonist, does not affect ER stress in human adipose tissue and adipocyte SGBS cell line [31], pioglitazone reduces ER stress in the liver of diet-induced diabetic mice [32]. Therefore, we also confirmed that ER stress was not alleviated by ciglitazone (Fig. 4C). Considering ciglitazone facilitates the transcriptional activity of PPAR- $\gamma$, these results suggested that the lipin-1 recovery by ciglitazone was due to increased lipin-1 transcription, not due to increased PPAR- $\gamma$ expression or decreased ER stress. The precise mechanism by which ciglitazone activates lipin-1 promoter needs to be validated in combination of several assays such as reporter assay and chromatin immunoprecipitation assay. 
Conversely, lipin-1 involves PPAR- $\gamma$ gene expression and functions.

Lipin-1 is required at an early step in adipocyte differentiation for the induction of the adipogenic gene transcription program, including the master regulator PPAR- $\gamma$ [4]. Lipin-1 works as a phosphatidic acid phosphatase to promote PPAR- $\gamma$ gene expression during adipogenesis by modulating phosphatidate levels [5]. Genetic analysis revealed that lipin-1 genetic variations affect the therapeutic responsiveness of a PPAR- $\gamma$ agonist rosiglitazone in type 2 diabetes [33].

The reduced adipose lipin-1 expression has been implicated in the pathogenesis of obesity, the metabolic syndrome, and type 2 diabetes [8-13]. Recently, we and the other group have showed that reduced adipose lipin-1 has been induced the expression of cytokines, MCP-1 and TNF- $\alpha$, suggesting adipose lipin-1 regulates inflammation $[7,15]$. Recovering the reduced lipin-1 expression in adipocytes could improve overall glucose metabolism, at least in part, by reducing these pro-inflammatory cytokines. Although, some factors such as TNF- $\alpha$, interleukin-1 $\beta$, and interferon- $\gamma$ have been revealed to reduce lipin-1 expression in adipocytes [34, 35], the relative contribution of ER stress to the lipin-1 depletion in adipocytes remains to be clarified.

In summary, we have demonstrated that ER stress is addressed as a new down-regulating factor for lipin-1 expression in adipocytes. Moreover, our present results suggest that PPAR- $\gamma$ may contribute to the constitutive expression of lipin-1 in adipocytes. Finally, activation of PPAR- $\gamma$ can alleviate the suppression of lipin-1 expression by ER stress. 
Acknowledgments: The authors thank the members of the Department of Biochemistry, School of Dentistry, Health Sciences University of Hokkaido, for the use of laboratory equipment. This work was supported, in parts by grants from the Ministry of Health, Labor and Welfare, Japan (M.I.), and Dainippon Sumitomo Pharmaceutical Co., Ltd. (N.T.). 


\section{References}

[1] M. Péterfy, J. Phan, P. Xu, K. Reue, Lipodystrophy in the fld mouse results from mutation of a new gene encoding a nuclear protein, lipin, Nat. Genet. 27 (2001) $121-124$.

[2] J. Donkor, M. Sariahmetoglu, J. Dewald, D.N. Brindley, K. Reue, Three mammalian lipins act as phosphatidate phosphatases with distinct tissue expression patterns, J. Biol. Chem. 282 (2007) 3450-3457.

[3] G.S. Han, The Saccharomyces cerevisiae lipin homolog is a $\mathrm{Mg}^{2+}$-dependent phosphatidate phosphatase enzyme, J. Biol. Chem. 281 (2006) 9210-9218. [4] Y.K. Koh, M.Y. Lee, J.W. Kim, M. Kim, J.S. Moon, Y.J. Lee, Y.H. Ahn, K.S. Kim, Lipin1 is a key factor for the maturation and maintenance of adipocytes in the regulatory network with CCAAT/enhancer-binding protein alpha and peroxisome proliferator-activated receptor $\gamma 2$, J. Biol. Chem. 283 (2008) 34896-34906. [5] P. Zhang, K. Takeuchi, L.S. Csaki, K. Reue, Lipin-1 phosphatidic phosphatase activity modulates phosphatidate levels to promote peroxisome proliferator-activated receptor $\gamma($ PPAR $\gamma)$ gene expression during adipogenesis, J. Biol. Chem. 287 (2012) $3485-3494$.

[6] B.N. Finck, M.C. Gropler, Z. Chen, T.C. Leone, M.A. Croce, T.E. Harris, J.C. Lawrence, Jr., D.P. Kelly, Lipin 1 is an inducible amplifier of the hepatic PGC-1alpha/PPARalpha regulatory pathway, Cell Metab. 4 (2006) 199-210.

[7] H.B. Kim, A. Kumar, L. Wang, G.H. Liu, S.R. Keller, J.C. Lawrence, Jr., B.N. Finck, T.E. Harris, Lipin 1 represses NFATc4 transcriptional activity in adipocytes to inhibit secretion of inflammatory factors, Mol. Cell. Biol. 30 (2010) 3126-3139. 
[8] E. Suviolahti, K. Reue, R.M. Cantor, J. Phan, M. Gentile, J. Naukkarinen, A. Soro-Paavonen, L. Oksanen, J. Kaprio, A. Rissanen, V. Salomaa, K. Kontula, M.R. Taskinen, P. Pajukanta, L. Peltonen, Cross-species analyses implicate lipin 1 involvement in human glucose metabolism, Hum. Mol. Genet. 15 (2006) 377-386. [9] Y.C. Chang, L.Y. Chang, T.J. Chang, Y.D. Jiang, K.C. Lee, S.S. Kuo, W.J. Lee, L.M. Chuang, The associations of LPIN1 gene expression in adipose tissue with metabolic phenotypes in the Chinese population, Obesity 18 (2010) 7-12. [10] J. Donkor, L.M. Sparks, H. Xie, S.R. Smith, K. Reue, Adipose tissue lipin-1 expression is correlated with peroxisome proliferator-activated receptor alpha gene expression and insulin sensitivity in healthy young men, J. Clin. Endocrinol. Metab. 93 (2008) 233-239.

[11] A. Yao-Borengasser, N. Rasouli, V. Varma, L.M. Miles, B. Phanavanh, T.N. Starks, J. Phan, H.J. Spencer, 3rd, R.E. McGehee, Jr., K. Reue, P.A. Kern, Lipin expression is attenuated in adipose tissue of insulin-resistant human subjects and increases with peroxisome proliferator-activated receptor gamma activation, Diabetes 55 (2006) 2811-2818.

[12] V. van Harmelen, M. Ryden, E. Sjolin, J. Hoffstedt, A role of lipin in human obesity and insulin resistance: relation to adipocyte glucose transport and GLUT4 expression, J. Lipid Res. 48 (2007) 201-206.

[13] M.A. Croce, J.C. Eagon, L.L. LaRiviere, K.M. Korenblat, S. Klein, B.N. Finck, Hepatic lipin 1 expression is diminished in insulin-resistant obese subjects and is reactivated by marked weight loss, Diabetes 56 (2007) 2395-2399.

[14] J. Phan, K. Reue, Lipin, a lipodystrophy and obesity gene, Cell Metab. 1 (2005) $73-83$. 
[15] N. Takahashi, T. Yoshizaki, N. Hiranaka, T. Suzuki, T. Yui, M. Akanuma, K.

Oka, K. Kanazawa, M. Yoshida, S. Naito, M. Fujiya, Y. Kohgo, M. Ieko, Suppression of lipin-1 expression increases monocyte chemoattractant protein-1 expression in 3T3-L1 adipocytes, Biochem. Biophys. Res. Commun. 415 (2011) 200-205.

[16] M. Cnop, F. Foufelle, L.A. Velloso, Endoplasmic reticulum stress, obesity and diabetes, Trends Mol. Med. 18 (2012) 59-68.

[17] U. Ozcan, Q. Cao, E. Yilmaz, A.H. Lee, N.N. Iwakoshi, E. Ozdelen, G.

Tuncman, C. Gorgun, L.H. Glimcher, G.S. Hotamisligil, Endoplasmic reticulum stress links obesity, insulin action, and type 2 diabetes, Science 306 (2004) 457-461. [18] M.F. Gregor, L. Yang, E. Fabbrini, B.S. Mohammed, J.C. Eagon, G.S. Hotamisligil, S. Klein, Endoplasmic reticulum stress is reduced in tissues of obese subjects after weight loss, Diabetes 58 (2009) 693-700.

[19] N.K. Sharma, S.K. Das, A.K. Mondal, O.G. Hackney, W.S. Chu, P.A. Kern, N. Rasouli, H.J. Spencer, A. Yao-Borengasser, S.C. Elbein, Endoplasmic reticulum stress markers are associated with obesity in nondiabetic subjects, J. Clin. Endocrinol. Metab. 93 (2008) 4532-4541.

[20] G. Boden, X. Duan, C. Homko, E.J. Molina, W. Song, O. Perez, P. Cheung, S. Merali, Increase in endoplasmic reticulum stress-related proteins and genes in adipose tissue of obese, insulin-resistant individuals, Diabetes 57 (2008) 2438-2444. [21] J. Vendrell, E. Maymo-Masip, F. Tinahones, A. Garcia-Espana, A. Megia, E. Caubet, E. Garcia-Fuentes, M.R. Chacon, Tumor necrosis-like weak inducer of apoptosis as a proinflammatory cytokine in human adipocyte cells: up-regulation in severe obesity is mediated by inflammation but not hypoxia, J. Clin. Endocrinol. 
Metab. 95 (2010) 2983-2992.

[22] N. Hosogai, A. Fukuhara, K. Oshima, Y. Miyata, S. Tanaka, K. Segawa, S. Furukawa, Y. Tochino, R. Komuro, M. Matsuda, I. Shimomura, Adipose tissue hypoxia in obesity and its impact on adipocytokine dysregulation, Diabetes 56 (2007) 901-911.

[23] P. Jiao, J. Ma, B. Feng, H. Zhang, J.A. Diehl, Y.E. Chin, W. Yan, H. Xu, FFA-induced adipocyte inflammation and insulin resistance: involvement of ER stress and IKKbeta pathways, Obesity (Silver Spring) 19 (2011) 483-491.

[24] K. Miyokawa-Gorin, K. Takahashi, K. Handa, A. Kitahara, Y. Sumitani, H. Katsuta, T. Tanaka, S. Nishida, K. Yoshimoto, H. Ohno, H. Ishida, Induction of mitochondrial uncoupling enhances $\mathrm{VEGF}_{120}$ but reduces $\mathrm{MCP}-1$ release in mature 3T3-L1 adipocytes: possible regulatory mechanism through endogenous ER stress and AMPK-related pathways, Biochem. Biophys. Res. Commun. 419 (2012) $200-205$.

[25] M.I. Lefterova, S.E. Mullican, T. Tomaru, M. Qatanani, M. Schupp, M.A. Lazar, Endoplasmic reticulum stress regulates adipocyte resistin expression, Diabetes 58 (2009) 1879-1886.

[26] M. Miranda, X. Escote, V. Ceperuelo-Mallafre, A. Megia, E. Caubet, S. Naf, J.M. Gomez, J.M. Gonzalez-Clemente, V. Vicente, J. Vendrell, Relation between human LPIN1, hypoxia and endoplasmic reticulum stress genes in subcutaneous and visceral adipose tissue, Int. J. Obes. (Lond) 34 (2010) 679-686.

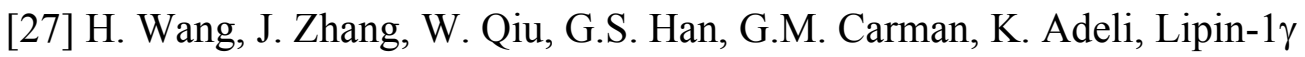
isoform is a novel lipid droplet-associated protein highly expressed in the brain, FEBS Lett. 585 (2011) 1979-1984. 
[28] M. Peterfy, J. Phan, K. Reue, Alternatively spliced lipin isoforms exhibit distinct expression pattern, subcellular localization, and role in adipogenesis, J. Biol. Chem. 280 (2005) 32883-32889.

[29] D. Ryu, W.Y. Seo, Y.S. Yoon, Y.N. Kim, S.S. Kim, H.J. Kim, T.S. Park, C.S. Choi, S.H. Koo, Endoplasmic reticulum stress promotes LIPIN2-dependent hepatic insulin resistance, Diabetes 60 (2011) 1072-1081.

[30] P. Zhang, L. O'Loughlin, D.N. Brindley, K. Reue, Regulation of lipin-1 gene expression by glucocorticoids during adipogenesis, J. Lipid Res. 49 (2008) $1519-1528$.

[31] S.K. Das, W.S. Chu, A.K. Mondal, N.K. Sharma, P.A. Kern, N. Rasouli, S.C. Elbein, Effect of pioglitazone treatment on endoplasmic reticulum stress response in human adipose and in palmitate-induced stress in human liver and adipose cell lines, Am. J. Physiol. Endocrinol. Metab. 295 (2008) E393-E400.

[32] K. Yoshiuchi, H. Kaneto, T. Matsuoka, R. Kasami, K. Kohno, T. Iwawaki, Y. Nakatani, Y. Yamasaki, I. Shimomura, M. Matsuhisa, Pioglitazone reduces ER stress in the liver: Direct monitoring of in vivo ER stress using ER stress-activated indicator transgenic mice, Endocr. J. 56 (2009) 1103-1111.

[33] E.S. Kang, S.E. Park, S.J. Han, S.H. Kim, C.M. Nam, C.W. Ahn, B.S. Cha, K.S. Kim, H.C. Lee, LPIN1 genetic variation is associated with rosiglitazone response in type 2 diabetic patients, Mol. Genet. Metab. 95 (2008) 96-100.

[34] B. Lu, Y. Lu, A.H. Moser, J.K. Shigenaga, C. Grunfeld, K.R. Feingold, LPS and proinflammatory cytokines decrease lipin-1 in mouse adipose tissue and 3T3-L1 adipocytes, Am. J. Physiol. Endocrinol. Metab. 295 (2008) E1502-1509. [35] Y. Tsuchiya, N. Takahashi, T. Yoshizaki, S. Tanno, M. Ohhira, W. Motomura, 
K. Takakusaki, Y. Kohgo, T. Okumura, A Jak2 inhibitor, AG490, reverses lipin-1 suppression by TNF- $\alpha$ in 3T3-L1 adipocytes, Biochem. Biophys. Res. Commun. 382 (2009) 348-352. 


\section{Figure legends}

Figure 1. Endoplasmic reticulum stress suppresses lipin-1 expression in 3T3-L1 adipocytes. Lipin-1 mRNA expression levels in 3T3-L1 adipocytes were measured after the treatment with different concentrations of tunicamycin (Tun) (A) or thapsigargin (Thap) (B) for $24 \mathrm{~h}$. Lipin-1 mRNA expression levels were measured before and after exposure of tunicamycin (Tun, $3 \mu \mathrm{g} / \mathrm{ml}$ ) or thapsigargin (Thap, 50 $\mathrm{nM}$ ) (C). 3T3-L1 adipocytes were treated with tunicamycin (Tun, $3 \mu \mathrm{g} / \mathrm{ml}$ ) or thapsigargin (Thap, $50 \mathrm{nM}$ ) for $24 \mathrm{~h}$. Then, lipin-1 protein expression was determined by Western blotting with a $\beta$-actin probe used as the loading control (D), and mRNA expression of lipin-1A and lipin-1B (E) or lipin-2 (F) was quantified by real-time PCR. Results are expressed as means $\pm \mathrm{SE}(n=3) .{ }^{*} P<0.05$ vs. vehicle (DMSO, Veh) (A, B, E, F) or time $0(\mathrm{C}) . \dagger P<0.05$ vs. lipin-1A in the same treatment group (E).

Figure 2. Messenger RNA stability is not involved in the thapsigargin-induced lipin-1 mRNA reduction in 3T3-L1 adipocytes. 3T3-L1 adipocytes were treated with $5 \mu \mathrm{g} / \mathrm{ml}$ of actinomycin D (ActD) in combination with thapsigargin (Thap, $50 \mathrm{nM}$ ) or vehicle (DMSO). Lipin-1 mRNA expression levels were measured by quantitative real-time PCR at times of 0,4 , and $24 \mathrm{~h}$. Results are calculated as fold change relative to the values at time 0 and expressed as means $\pm \operatorname{SE}(n=3)$. No significant difference was observed in both treatment groups. 
Figure 3. Endoplasmic reticulum stress reduces $\mathrm{C} / \mathrm{EBP}-\alpha$ and PPAR- $\gamma$ expression, and knockdown of PPAR- $\gamma$ expression results in the reduction of lipin-1 expression in 3T3-L1 adipocytes. 3T3-L1 adipocytes were treated with tunicamycin (Tun, 3 $\mu \mathrm{g} / \mathrm{ml}$ ) or thapsigargin (Thap, $50 \mathrm{nM}$ ) for $24 \mathrm{~h}$. Protein expression of C/EBP- $\alpha$ and PPAR- $\gamma$ was analyzed by Western blotting with a $\beta$-actin probe used as the loading control (A). Messenger RNA expression levels of C/EBP- $\alpha$ (B), and PPAR- $\gamma$ (C) were quantified by real-time PCR. DMSO was used as vehicle (Veh). Next, 3T3-L1 adipocytes were transfected with either C/EBP- $\alpha$ or PPAR- $\gamma$ specific siRNA at a final concentration of $50 \mathrm{nM}$ for $60 \mathrm{~h}$. Negative control siRNA (si-Neg) was used as vehicle. Knockdown of the gene expression of C/EBP- $\alpha$ (si-C/EBP- $\alpha \mathrm{D})$ or PPAR- $\gamma$ (si-PPAR- $\gamma$, E) was confirmed by real-time PCR. Then, lipin-1 mRNA expression levels were determined in either C/EBP- $\alpha$ or PPAR- $\gamma$ knocked down adipocytes compared with the vehicle (F). Results are expressed as means $\pm \operatorname{SE}(n=3)$. ${ }^{*} P<$ 0.05 vs. vehicle.

Figure 4. Ciglitazone recovers thapsigargin-induced lipin-1 depletion in 3T3-L1 adipocytes. 3T3-L1 adipocytes were treated with thapsigargin (Thap, $50 \mathrm{nM}$ ) or vehicle (DMSO, Veh) for $24 \mathrm{~h}$. At the last $8 \mathrm{~h}$ of the treatment, ciglitazone (Cig, 30 $\mu \mathrm{M})$ or vehicle was added to the medium. Lipin-1 mRNA expression levels were quantified by real-time PCR (A). Results are expressed as means $\pm \operatorname{SE}(n=3)$. ${ }^{*} P<$ 0.05 vs. vehicle. Protein expression levels of lipin-1 (B), PPAR- $\gamma, \mathrm{BiP}$, and CHOP (C) were visualized by Western blotting with a $\beta$-actin probe used as the loading control. 


\section{Supplementary Figure}

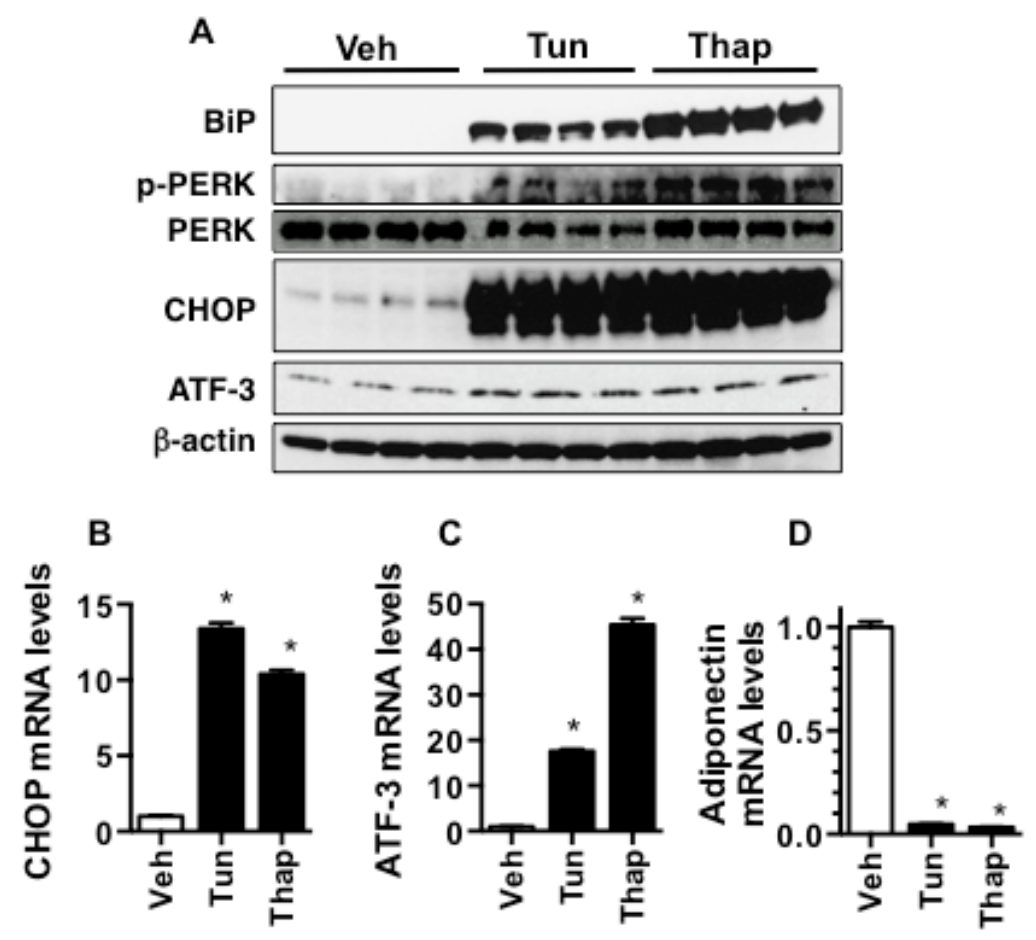

Figure S1. Tunicamycin and thapsigargin induce endoplasmic reticulum (ER) stress in 3T3-L1 adipocytes. Fully differentiated 3T3-L1 adipocytes were treated with tunicamycin (Tun, $3 \mu \mathrm{g} / \mathrm{ml}$ ) or thapsigargin (Thap, $50 \mathrm{nM}$ ) for $24 \mathrm{~h}$. DMSO was used as vehicle (Veh). Unfolded protein response was assessed by Western blotting and quantitative real-time PCR (TaqMan gene expression assay, Applied Biosystems, Foster City, CA, USA). Protein expression of BiP, phospho-PERK, PERK, CHOP (Cell Signaling Technology, Danvers, MA, USA), and ATF-3 (Bioworld technology, St. Louis Park, MN, USA), were determined by Western blotting with a $\beta$-actin probe used as the loading control (A). Messenger RNA levels of CHOP (Mm00492097_m1, B), ATF-3 (Mm00476032_m1, C), and adiponectin 
(Mm00456425_m1, D) were determined, subsequently normalized relative to mRNA levels of 18S rRNA and expressed as fold change relative to vehicle. Results are presented as means \pm SE $(n=3) . * P<0.05$ vs. vehicle. Unfolded protein response was strongly induced by the treatment with tunicamycin and thapsigargin (A-C). The mRNA expression of adiponectin was reduced in 3T3-L1 adipocytes by the treatment with both ER stress inducers (D), which is in agreement with a previous report [1]. These results documented that ER stress is induced by the treatment with tunicamycin and thapsigargin in 3T3-L1 adipocytes.

[1] N. Hosogai, A. Fukuhara, K. Oshima, Y. Miyata, S. Tanaka, K. Segawa, S. Furukawa, Y. Tochino, R. Komuro, M. Matsuda, I. Shimomura, Adipose tissue hypoxia in obesity and its impact on adipocytokine dysregulation, Diabetes 56 (2007) 901-911. 

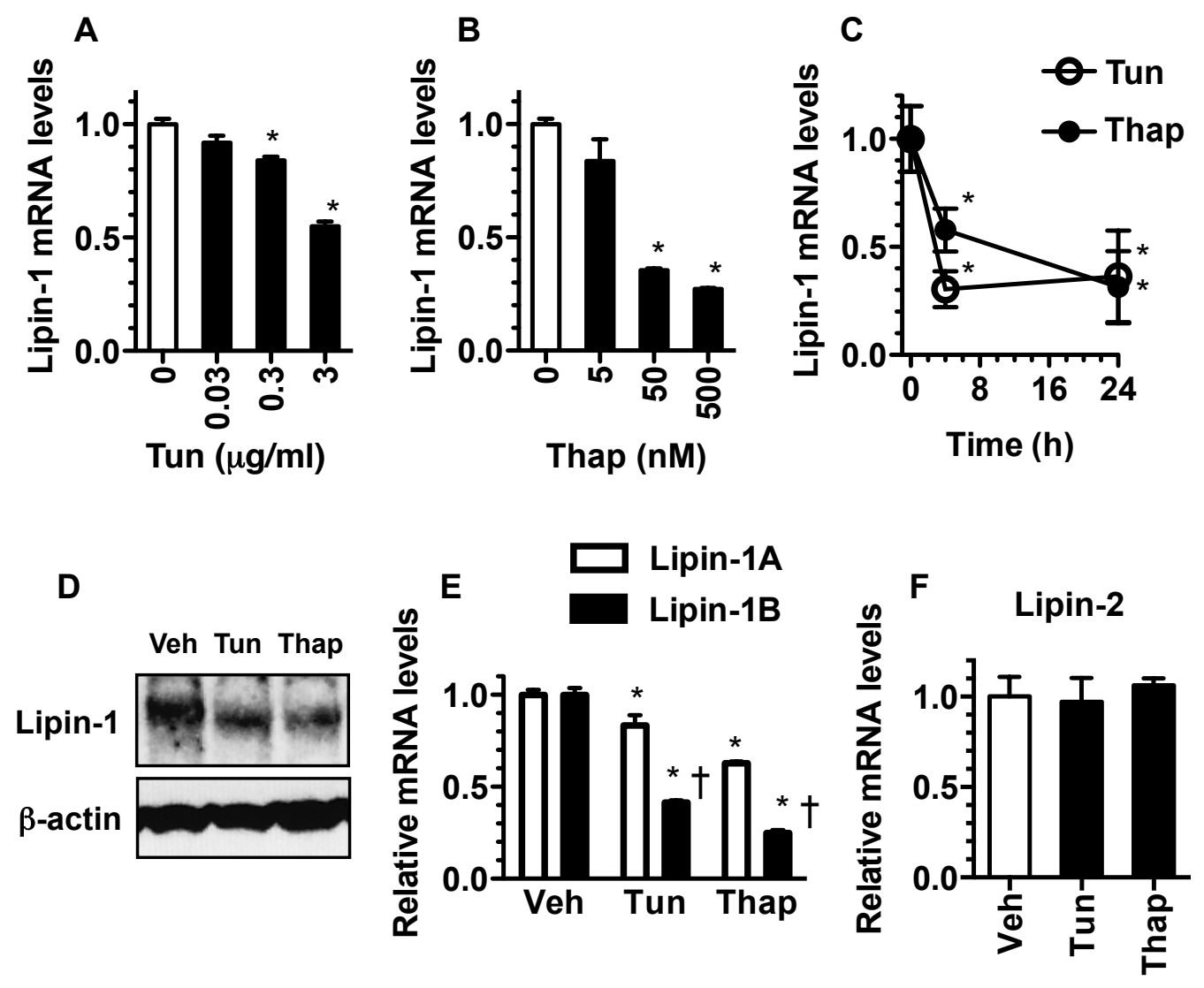


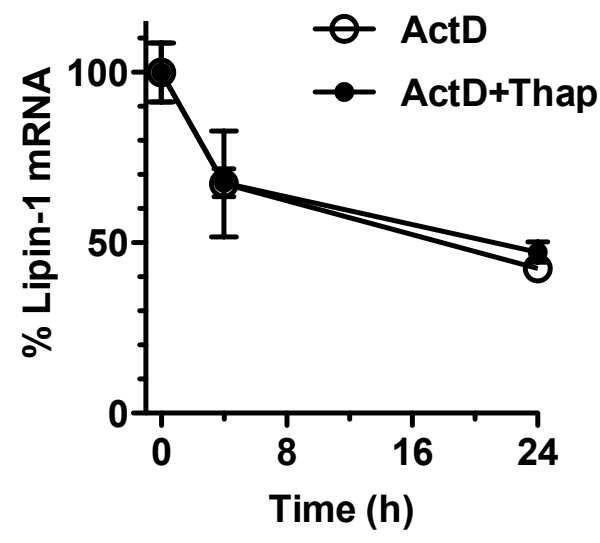



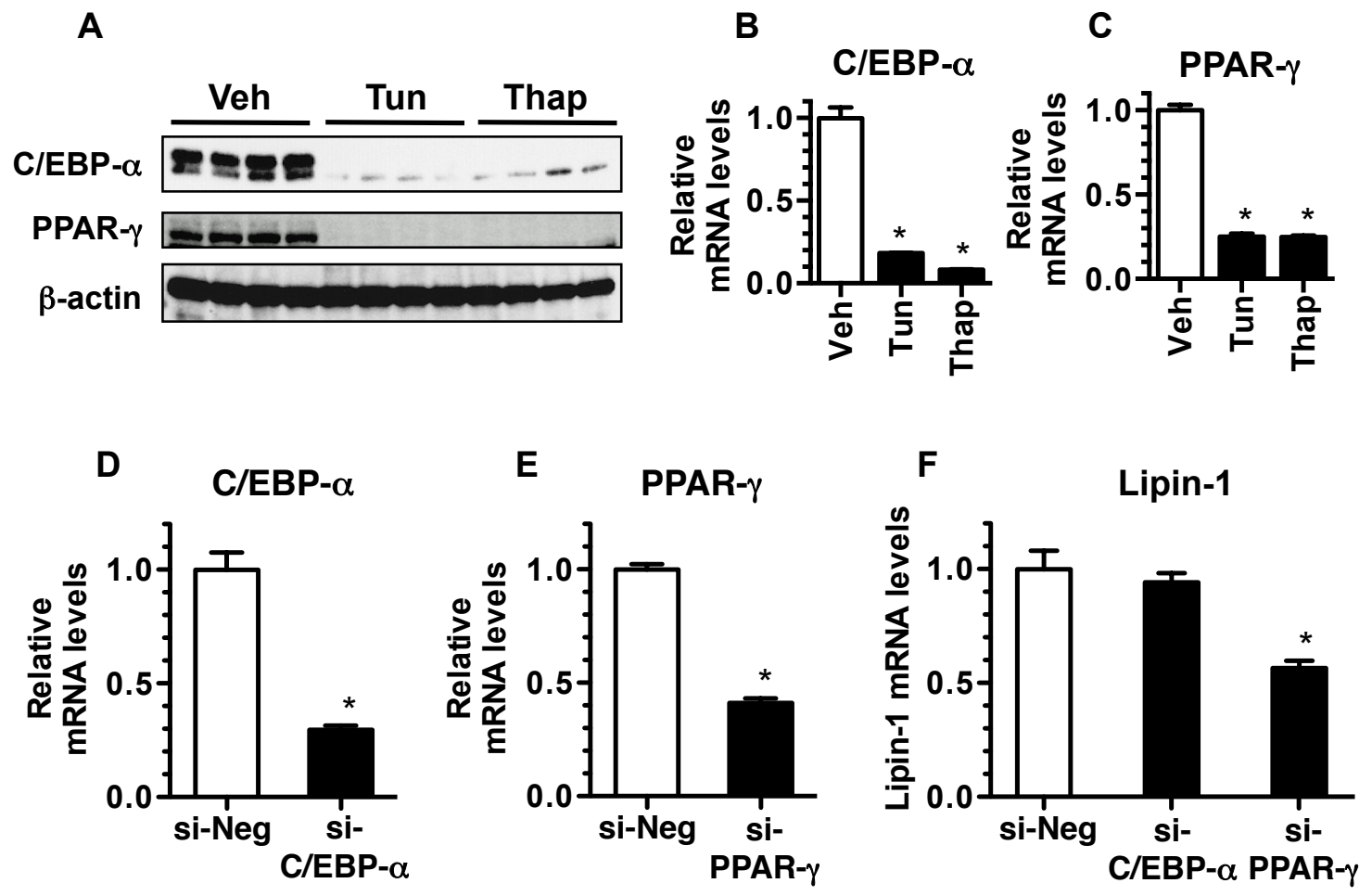


\section{A Lipin-1}

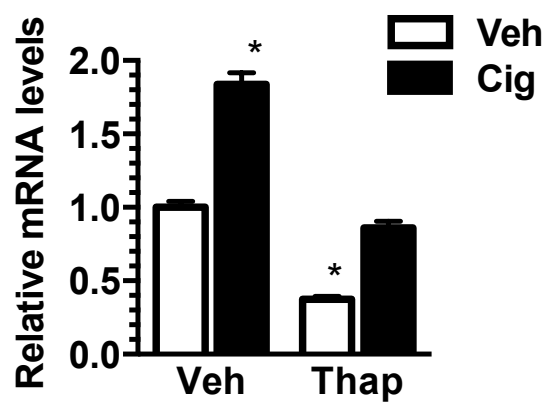

B

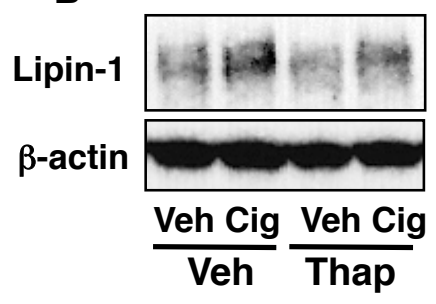

C

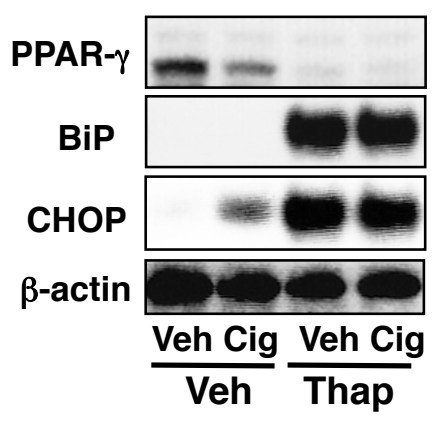

